\title{
Lipid-coated nanowires enable small-scale bioelectronics
}

\author{
Aleksandr Noy, Nipun Misra, and Julio Martinez
}

Integration of biological molecules into nanoelectronic devices promises new, sophisticated biointerfacing technologies.

Ever since x-ray diffraction first revealed protein structures, biological molecules have fascinated scientists with their perfectly engineered, elegant designs able to perform functions well beyond the reach of conventional devices. Inevitably, researchers have dreamed of developing a functional interface between living organisms and electronic circuits, enabling better, more specific diagnostics and prosthetics that could be controlled by direct input from the peripheral nervous system or the brain.

The idea of using microelectronics to probe the activity of biological systems originated with early work on capacitive stimulation of cells. ${ }^{1}$ More recently, neuronal control of siliconnanowire (SiNW) transistor arrays has been demonstrated. ${ }^{2}$ Yet the clear mismatch in scale between cellular machinery and electronic circuitry made the early interfaces crude and inefficient. The discovery of carbon nanotubes and SiNWs has given researchers a new opportunity to construct electronic interfaces comparable in size to biological molecules. We have shown how the electrical response of a nanowire transistor can be controlled by an ion-channel protein placed in close proximity to the nanowire, and even that voltage applied by the device can control those biological channels.

Our versatile, self-assembled nanoplatform integrates a fieldeffect transistor (FET) with membrane-bound proteins that mediate the majority of signaling in living systems. ${ }^{3}$ A cell membrane - its outer layer-is made up of phospholipids, molecules with a hydrophilic head and two hydrophobic tails that arrange themselves into a two-layered sheet (bilayer) with their tails pointing inwards when exposed to water. Proteins embedded in the membrane can act as 'pores' or channels, allowing certain signaling ions to pass through the cell's outer layer. Inhibitors can block the pore and prevent other ions from passing through. Our nanoplatform is a 1D lipid-bilayer membrane covering a nanowire template. ${ }^{4}$ The lipid bilayer closely mimics a
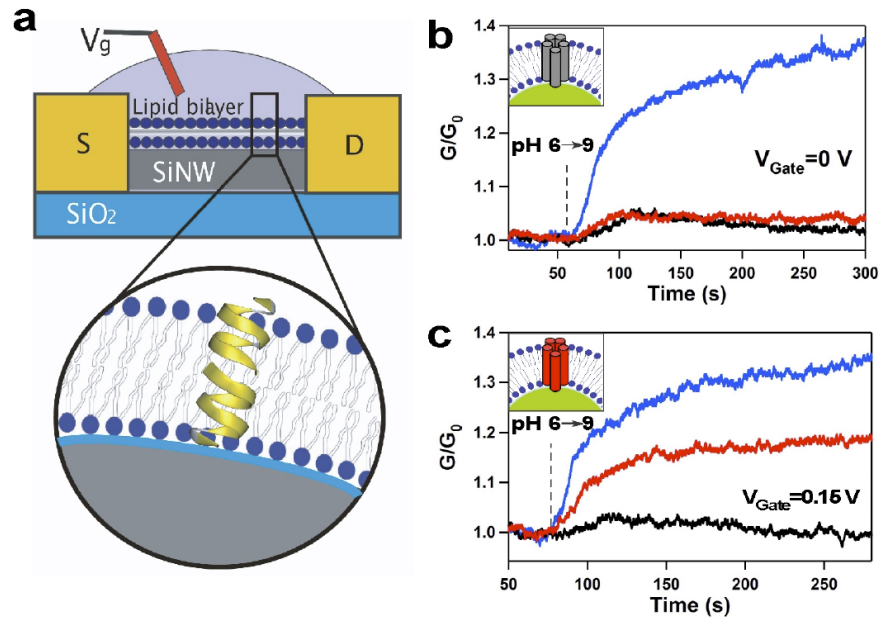

Figure 1. (a) Device schematics showing an ion channel embedded in the lipid bilayer covering the silicon nanowire (SiNW). S and D denote source and drain electrodes and $V_{g}$ is the applied gate voltage. (b) SiNW normalized conductance, $G / G_{0}$, recorded as the solution's pH was changed from 6 to 9 for the uncoated (blue) and coated NW (black), and the coated-NW device incorporating Alamethicin pores (red). (c) Time traces of a similar experiment recorded at a gate bias of 0.15V. (C) 2009 The National Academy of Sciences. Reproduced with permission..$\left.^{5}\right)$

cellular membrane, creating an impermeable barrier around the wire, yet this barrier can incorporate membrane proteins.

We recently demonstrated some of the first examples of such integration. ${ }^{5}$ In our FET-based devices, a small semiconducting SiNW is connected to a pair of metallic source and drain electrodes passivated with a layer of silicon nitride, $\mathrm{Si}_{3} \mathrm{~N}_{4}$ (the passivation layer protects and insulates the electrodes from the buffer solution used for the experiments with biological molecules): see Figure 1. FETs allow current to pass between source and drain electrodes when a suitable voltage is applied to a 'gate' between them. The key step was the formation of the lipid bilayer on the surface of the SiNW using the native silicon oxide as a template. 
In our self-assembly procedure, lipid vesicles, small spheres of lipid membrane, fuse on the nanowire surface, forming a continuous coating.

We used measurements of cyclic voltammetry to show that formation of the lipid bilayer on the nanowire surface reduces the limiting current by $85-95 \%$ relative to the uncoverednanowire device, demonstrating that the bilayer provided an effective shield that insulates the nanowire surface from the surrounding solution. As a detection mechanism, our devices exploited the sensitivity of the nanowire surface to the solution's $\mathrm{pH}$. Charging of silanol groups at the silicon oxide layer on the surface of SiNWs is known to change the source-drain current at a given gate voltage. ${ }^{6}$ Indeed, bare nanowire FETs showed a pronounced increase in conductance when the $\mathrm{pH}$ of their fluidic environment was changed from six to nine: see Figures 1(b) and 1(c). As expected, lipid-bilayer formation on the SiNW surface led to a sharp decrease in the FET response to $\mathrm{pH}$ changes in the fluid cell.

When we incorporated Gramicidin A protein pores, we obtained a dramatic recovery of the device's $\mathrm{pH}$ response, indicating formation of ion channels in the lipid shell. Moreover, this response could be modulated by adding a $1 \mathrm{mM}$ solution of calcium ions, a well-known Gramicidin A inhibitor. This measurement demonstrates that a bioelectronic device can successfully convert a chemical ( $\mathrm{pH}$ change) into an electrical signal, and that such a device can be regulated by the same ligandgating mechanism as used in biological systems.

Finally, we showed that the nanoelectronic device could control the output of a biological ion channel. When the nanowire devices incorporated a voltage-gated peptide Alamethicin (ALM), we could use the electric field of the device to open and close the peptide pore. At zero applied gate bias, ALM pores were 'turned off' and the device showed little response to $\mathrm{pH}$ changes: see Figure 1(b). However, at a positive gate voltage of $150 \mathrm{mV}$, it showed a strong response to $\mathrm{pH}$ changes, indicating that the ALM pores had been 'turned on.' These results demonstrate that our device platform is not only capable of using biological components as functional parts, but can also control the functionality of biological molecules.

In summary, these devices represents a universal platform for building bio/nano interfaces. We have used SiNW devices and proton transport for proof-of-concept measurements, showing that we can control the electrical current across a transistor through its chemical environment and biological ion channels and also manipulate those ion channels using an applied voltage. However, other ion-sensitive device designs do not have to be restricted to these components. The lipid bilayers provide a matrix for a virtually unlimited number of membrane proteins with diverse functions and these biomimetic devices could power many applications in biosensing and bioelectronics. Some likely next steps include the use of more sophisticated biological machines, for example proteins powered by adenosine triphosphate, in the nanoelectronic device.

\section{Author Information}

Aleksandr Noy, Julio Martinez, and Nipun Misra

Physical and Life Sciences Directorate

Lawrence Livermore National Laboratory (LLNL)

Livermore, CA

Aleksandr Noy joined LLNL as an E. O. Lawrence fellow after completing his doctoral work at Harvard University. He became a staff scientist in 2001 and also serves as an adjunct professor at the University of California, Merced. His research interests are in the broad area at the intersection of biophysics and nanotechnology, centering on 1D materials.

Julio Martinez received his PhD from the University of California, Davis, in 2009. Part of his doctoral work was undertaken with Aleksandr Noy and colleagues at LLNL. He currently works as a postdoctoral researcher at the Sandia National Laboratory in Albuquerque (NM).

Nipun Misra received his PhD from University of California, Berkeley, in 2009. Part of his doctoral work was undertaken with Aleksandr Noy and colleagues at LLNL. He currently works at Applied Materials Inc. in Santa Clara (CA).

\section{References}

1. P. Fromherz and A. Stett, Silicon-neuron junction: capacitive stimulation of an individual neuron on a silicon chip, Phys. Rev. Lett. 75, p. 1670, 1995.

2. F. Patolsky et al., Detection, stimulation, and inhibition of neuronal signals with highdensity nanowire transistor arrays, Science 313, p. 1100, 2006.

3. B. Alberts et al., Molecular Biology of the Cell, 3rd ed., Garland, NY, 1994.

4. A. B. Artyukhin et al., Functional one-dimensional lipid bilayers on carbon nanotube templates, J. Am. Chem. Soc. 127, p. 7538, 2005.

5. N. Misra et al., Bioelectronic silicon nanowire devices utilizing functional membrane proteins, Proc. Nat'1 Acad. Sci. USA 106, p. 13780, 2009.

6. Y. Cui, Q. Q. Wei, H. K. Park, and C. M. Lieber, Nanowire nanosensors for highly sensitive and selective detection of biological and chemical species, Science 293, p. 1289, 2001. 